\title{
ON THE BEST CHOICE OF A DAMPING SEQUENCE IN ITERATIVE OPTIMIZATION METHODS
}

\author{
L.N. VASERSTEIN
}

\begin{abstract}
Some iterative methods of mathematical programming use a damping sequence $\left\{\alpha_{2}\right\}$ such that $0 \leq \alpha_{t} \leq I$ for all $t, \alpha_{t} \rightarrow 0$ as $t \rightarrow \infty$, and $\sum \alpha_{t}=\infty$. For example, $\alpha_{t}=1 /(t+1)$ in Brown's method for solving matrix games. In this paper, for a model class of iterative methods, the convergence rate for any damping sequence $\left\{\alpha_{t}\right\}$ depending only on time $t$ is computed. This computation is used to find the best damping sequence.
\end{abstract}

\section{Iterative procedure}

Let $L$ be a real affine space (so $L$ with an origin fixed is the same as a real vector space). For any points $x$ and $y$ in $L$, let $[x, y]$ denote the closed interval with the ends $x$ and $y$. For any real-valued function $f$ on a subset $X$ of $L$, let $f(X)$ denote its infimum on $X$.

On a non-empty subset $X$ of $L$, we consider an iterative procedure of the form

$$
x_{t}=\left(1-\alpha_{t}\right) x_{t-1}+\alpha_{t} y_{t}=x_{t-1}+\alpha_{t}\left(y_{t}-x_{t-1}\right),
$$

where $0 \leq \alpha_{t} \leq 1,1 \leq t<T+1$, and $\left[y_{t}, x_{t-1}\right] \subset X$.

Here the total number $T$ of iterations is either finite or infinite $(T=\infty)$; in the second case $t$ runs over all natural numbers.

The objective of the procedure, starting at a point $x_{0}$ of $X$, is to minimize a convex bounded from below function $f$ on $X$. (We call $f$ convex on $X$, if its restriction on every interval contained in $X$ is convex).

To reach this objective, at each step $t$, one tends to choose $y_{t}$ in $X$, so that $f$ decreases when one starts to move from $x_{t-1}$ to $y_{t}$. The choice of $y_{t}$ depends, in general, on $f, x_{t-1}$, and $t$. We abstract ourselves from any concrete rule of choosing $y_{t}$, and just assume that the choice was good enough. Namely, we fix a number $\theta$ in the interval $0 \leq \theta<1$ and consider the class of iterative methods such that

$$
f\left(\left[x_{t-1}, y_{\ell}\right]\right)-f(X) \leq \theta\left(f\left(x_{t-1}\right)-f(X)\right)
$$


for all integers $t$ in the interval $1 \leq t<T+1$.

Note that according to (1), after a good direction $y_{t}-x_{t-1}$ is chosen, we do note minimize $f$ on the interval $\left[x_{t-1}, y_{t}\right]$, but make a step from $x_{t-1}$ in direction to $y_{t}$, with a "stepsize" $\alpha_{t}$ depending only on $t$.

Iterative procedures of the form (1) can be used not only for minimization of convex functions (see, for example, [4]). Sometimes they can be used for minimization of a not necessary convex bounded from below function $g$ on $X$, because, for an arbitrary $g$, its infimum $g(X)$ is equal to $f(X)$, where $f$ is the largest convex function on $X$ such that $f \leq g$ everywhere on $X$. This $f$ exists for any $g$, because the supremum of any set of convex functions on an interval is convex. This approach is feasible, if directions satisfying the condition (2) can be easily chosen.

Also the procedures of the form (1) can be used to search for a convex subset $X_{\infty}$ of $X$. For example, this $X_{\infty}$ could be a point where a function on $X$ reaches a critical value. The search for $X_{\infty}$ can be reduced to minimization of a convex function $f$ as follows. Pick a distance $\rho$ on $L$ invariant under all translations and such that $\rho(x, x+(y-x) \alpha)=\rho(x, y) \alpha$ for all $x$ and $y$ in $L$ and all real numbers $\alpha \geq 0$. (So, when an origin 0 in $L$ is fixed, $(L, \rho(0, \cdot)$ ) is a linear normed space in the sense of Day [3].) Then $f=\rho\left(X_{\infty}, \cdot\right)$ is a convex non-regative function on $L$ and $X_{\infty}$ consists of the points which minimize $f$.

The condition (2) for such $f$ takes the form

$$
\rho\left(X_{\infty},\left[x_{t-1}, y_{t}\right]\right) \leq \theta \rho\left(X_{\infty}, x_{t-1}\right) \text { for all } t, 1 \leq t<T+1 .
$$

The distance $\rho(Y, Z)$ between two subsets of a metric space is defined to be the infimum of all $\rho(y, z)$, where $y \in Y$ and $z \in Z$.

Speaking of the convergence rate, minimization on the interval $\left[x_{t-1}, y_{t}\right]$ under the condition (2) would give the exponential convergence

$$
f\left(x_{\ell}\right)-f(X) \leq \theta^{t}\left(f\left(x_{0}\right)-f(X)\right) .
$$

Avoiding computation of stepsize (there is no line search in (1)), we will obtain (for the best damping sequence) a slower convergence

$$
f\left(x_{t}\right)-f(X) \leq C(1-\theta)^{-2} t^{-1} \text { (see Theorem } 3(b, c) \text { below). }
$$

One cannot get a better convergence assuming that $X$ is convex, and $f$ is a convex function defined on the whole $L$ (see the remarks in Sections 2 and 3 below).

Slow convergence of methods of the form (1) is sometimes compensated by their resistance to errors and data perturbations. The methods can be useful when data are uncertain and a precise solution is not feasible. See, for example, Belen'ky et al [1], where Robinson [5] result on the convergence of Brown's method [2] is generalized and applications to linear programming are given. 


\section{Convergence when a damping sequence is fixed}

We fix the total number $T \geq 1$ ( $T$ is an integer or $\infty$ ) of iterations, and a real number $F$. We impose the following condition on the function $f$ and the procedure (1):

$$
f\left(y_{t}\right)-f(X) \leq F \text { for } 0 \leq t<T+1 \text {, where } y_{0}=x_{0} .
$$

When $f$ is bounded from above (as well as from below) this condition holds automatically for a sufficiently large $F$.

Theorem 1. Fix $\theta$ in the interval $0 \leq \theta<1, T \leq \infty, F>0$, and a sequence $\left\{\alpha_{t}\right\} \in[0,1]^{T}$. Set $d_{0}:=1$ and

$$
d_{t}:=\max \left(d_{t-1}\left(1-\alpha_{t}+\alpha_{t} \theta\right), \theta d_{t-1}\left(1-\alpha_{t}\right)+\alpha_{t}\right), \quad 1 \leq t<T+1
$$

Then for any $L$ and $X$, any $f$ convex on $X$, and any iterative procedure (1) satisfying the conditions (2) and (4), we have

$$
f\left(x_{t}\right)-f(X) \leq F d_{t}, \quad 0 \leq t<T+1
$$

Moreover, there are $L, X, f$ as above and a procedure (1) satisfying (2), (4) such that for all $t$

$$
f\left(x_{t}\right)-f(X)=F d_{t}
$$

Proof: Note that a "procedure (1)" is determined by a starting point $x_{0}$ and a sequence $y_{t}$ in $X$ such that $\left[y_{t}, x_{t-1}\right] \subset X$, since the sequence $\left\{\alpha_{t}\right\}$ is fixed. We prove the first conclusion by induction on $t$. When $t=0, f\left(x_{0}\right)-f(X) \leq F d_{0}$ by $(4)$.

Let now $t \geq 1$ and we have proved that $f\left(x_{t-1}\right)-f(X) \leq F d_{t-1}$. The function $g(\alpha):=f\left(x_{t-1}+\alpha\left(y_{t}-x_{t-1}\right)\right)$ on the interval $[0,1]$ is convex and $g(0)=f\left(x_{t-1}\right) \leq f(X)+F d_{t-1}$. Moreover $g(1)=f\left(y_{t}\right) \leq f(X)+F$ by $(4)$ and $g([0,1]) \leq f(X)+\theta F d_{t-1}$ by $(2)$.

It follows that

$$
\begin{gathered}
f\left(x_{t-1}+\alpha\left(y_{t}-x_{t-1}\right)\right)=g(\alpha) \leq \\
\leq \max ((1-\alpha) g([0,1])+\alpha g(1),(1-\alpha) g(0)+\alpha g([0,1])) \leq \\
\leq f(X)+F_{\max }\left((1-\alpha) \theta d_{t-1}+\alpha,(1-\alpha) d_{t-1}+\alpha \theta d_{t-1}\right) \text { for all } \alpha .
\end{gathered}
$$


In particular, when $\alpha=\alpha_{t^{\prime}}$ we obtain our conclusion: $f\left(x_{t}\right)-f(X) \leq F d_{t}$. See Figure 1, where $f(X)=0$.

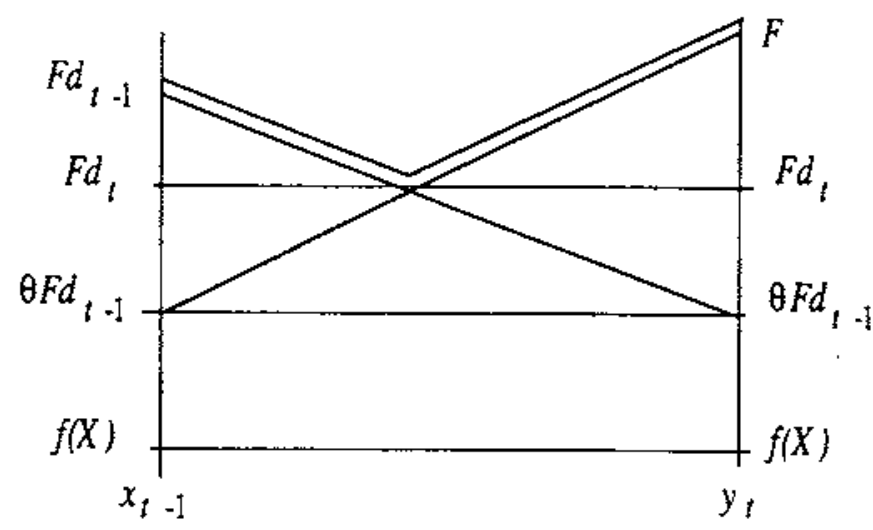

Figure 1: Upper bound for $f$ on the interval $\left[x_{t-1}, y_{t}\right]$

Now we want to construct $L, X, f$, and a procedure such that $f\left(x_{t}\right)-f(X)=$ $=F d_{t}$ for all $t$. Let $L$ be a $(T+1)$-dimensional real affine space, spanned by its $T+2$ points $y_{t},-1 \leq t<T+1$, in general position (so $y_{t}$ does not belong to the affine subspace spanned by all $y_{s}$ with $s<t$, where $-1<t<T+1$ ). Set $x_{0}:=y_{0}$ and $x_{t}:=x_{t-1}+\alpha_{t}\left(y_{t}-x_{t-1}\right)$ for $1 \leq t<T+1$. Set $X:=$ $\left\{y_{-1}\right\} \cup\left(\cup_{1 \leq t<T+1}\left[x_{t-1}, y_{t}\right]\right)$. Set $f\left(y_{-1}\right)=0$ and $f\left(y_{0}\right)=F$. For any $\alpha$ in the interval $0<\alpha \leq 1$ and any $t$ in the interval $0 \leq t<T+1$, we set

$$
f\left((1-\alpha) x_{t}+\alpha y_{t+1}\right)=\left\{\begin{array}{c}
(1-\alpha) \theta F d_{t}+F \text { when } \theta d_{t}\left(1-\alpha_{t+1}\right)+\alpha_{t+1} \geq \\
\geq d_{t}\left(1-\alpha_{t+1}(1-\alpha)\right), \\
\left((1-\alpha) d_{t}+\alpha \theta d_{t}\right) F \text { otherwise }
\end{array}\right.
$$

Then $f$ is a convex function on $X, f(X)=0$, and $f\left(x_{t}\right)=d_{t}$ for all $t$.

Remark. We could give a similar example with $X$ in plane $L$, see Figure 2. But the $(T+1)$-dimensional example above can be easily modified to an example with a convex $X$. Namely our function $f$ on $X$ can be extended to a convex function $f^{\prime}$ on the convex hull

$$
X^{\prime}=\left\{x \in L: x^{\prime}=\sum \gamma(x) x\right\}
$$

(here and below $\gamma$ ranges over all non-negative functions on $X$ taking only finitely many non-zero values and such that $\sum \gamma(x)=1$, so $\sum \gamma(x) x$ is a convex linear combination of points in $X$ ) of $X$ as follows:

$$
f^{\prime}\left(x^{\prime}\right):=\inf \left(\sum \gamma(x) f(x): \sum \gamma(x) x=x^{\prime}\right) .
$$




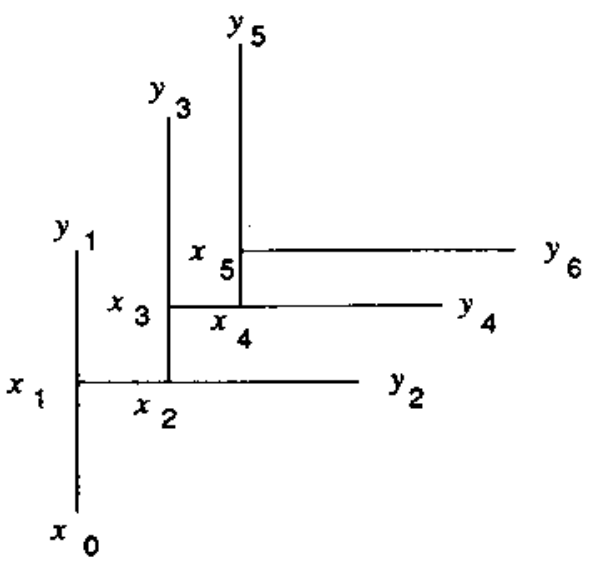

Figure 2 (see the remark)

At a small cost, an example with a convex $f$ defined on the whole $L$ can be constructed. Namely, for any $\varepsilon>0$ we can construct a convex function $f=f_{\varepsilon}$ on $L$ and a procedure (1), (2), (4) such that $f\left(x_{t}\right)-f(X) \geq F\left(d_{t}-\varepsilon\right)$ for all $t$. Indeed, let $L$ and $\left\{y_{t}\right\}_{-1 \leq t<T+1},\left\{x_{t}\right\}_{0 \leq t<T+1}$ be as above. We define $f$ on the line $R_{-1}=\left\{(1-\alpha) y_{-1}+\alpha y_{0}: \alpha\right.$ real $\}$ as follows: $f\left((1-\alpha) y_{-1}+\alpha y_{0}\right)=$ $\max (0, \alpha F)$. For any $t$ in the interval $0 \leq t<T+1$, we define a convex function $f$ on the line $R_{t}=\left\{(1-\alpha) x_{t}+\alpha y_{t+1}: \alpha\right.$ real $\}$ as follows.

When $\theta d_{t}\left(1-\alpha_{t+1}\right)+\alpha_{t+1} \geq d_{t}\left(1-\alpha_{t+1}(1-\theta)\right)$, we set $f\left((1-\alpha) x_{t}+\alpha y_{t+1}\right)=$ $\max \left(f\left(x_{t}\right)-\alpha f\left(x_{t}\right)(1-\theta)\left(F-\theta f\left(x_{t}\right)+\varepsilon F\right) /(\varepsilon F), F \alpha+(1-\alpha)\left(\theta f\left(x_{t}\right)-F \varepsilon\right)\right)$ (see Figure 3).

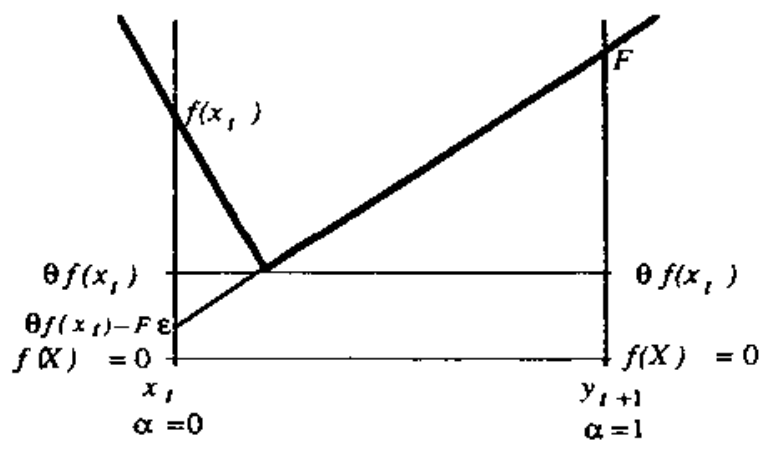

Figure 3 
Otherwise, we set $f\left((1-\alpha) x_{t}+\alpha y_{t+1}\right)=\max \left(((1-\alpha)+\alpha \theta) f\left(x_{t}\right), f\left(x_{t}\right) \theta\right)$ (see Figure 4).

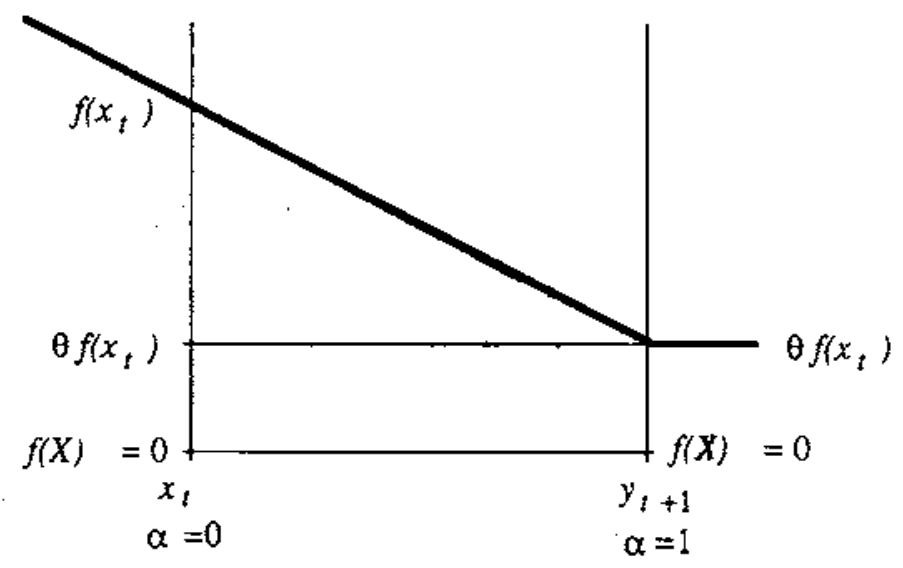

Figure 4

Then $\left|f\left(x_{t}\right)-F d_{t}\right| \leq F \varepsilon$ for all $t$. Now we can extend $f$ from the union $X=\cup_{0 \leq t<T+1} X_{t}$ of all lines $X_{t}$ to a convex function $f$ on the affine space $L$ spanned by the union. Replacing $X$ by its convex hull, we can arrange $X$ to be convex.

Theorem 2. Under the conditions of Theorem 1 assume that $T=\infty$. Set $\alpha_{\infty}:=\limsup \alpha_{t}$ and $d_{\infty}:=\limsup d_{t}$. Then:

(a) if $\sum_{t=1}^{\infty} \alpha_{t}=+\infty$, then $d_{\infty} \leq \frac{\alpha_{\infty}}{1-\theta+\alpha_{\infty} \theta}$;

in particular, when $\alpha_{\infty}=0$, i.e. $\alpha_{t} \rightarrow 0$, then $d_{\infty}=0$. i.e. $d_{t} \rightarrow 0$, i.e. $f\left(x_{i}\right) \rightarrow f(X)$ uniformly for all $L, X, f$ as above and all procedures (1), (2), (4) with fixed $\theta$ and $F$;

(b) if $\sum_{t=1}^{\infty} \alpha_{t}<\infty$, then $0<d_{\infty}=\sup _{0 \leq t<+\infty} d_{t} \Pi_{s=t+1}^{\infty}\left(1-\alpha_{s}+\alpha_{g} \theta\right)$;

(c) if $\alpha_{\infty}>0$, then $d_{\infty} \geq \alpha_{\infty}>0$; when, moreover, $\alpha_{t} \rightarrow \alpha_{\infty}>0$, then $d_{t} \rightarrow d_{\infty}=\alpha_{\infty} /\left(1-\theta+\alpha_{\infty} \theta\right)$;

(d) if $X$ contains an interval $[x, y]$ and a point $z$ strictly inside $[x, y]$ such that $f(x / 2, y / 2)=f(X) \neq f(z)$, then in the cases (b) and (c) there is a procedure (1), (2), (4) such that $f\left(x_{t}\right)$ does not converge to $f(X)$.

Proof: (a) We take any $\alpha$ such that $\alpha>\alpha_{t}$ for all $t>t_{0}$. We have to prove that $d_{\infty} \leq \alpha /(1-\theta+\alpha \theta)$. Let $t>t_{0}$.

If $d_{t} \geq \alpha /(1-\theta+\alpha \theta)$, then using that $\alpha>\alpha_{t+1}$ we conclude that

$$
d_{t} \geq \alpha_{t+1} /\left(1-\theta+\alpha_{t+1} \theta\right) \text {, i.e. } d_{t} \theta\left(1-\alpha_{t+1}\right)+\alpha_{t+1} \leq d_{t} .
$$

On the other hand, by the definition, $d_{t+1}:=\max \left(d_{t}\left(1-\alpha_{t+1}+\alpha_{t+1} \theta\right), \theta d_{t}\left(1-\alpha_{t+1}\right)+\alpha_{t+1}\right) \leq \theta d_{t}\left(1-\alpha_{t+1}\right)+\alpha_{t+1}$. 
So $d_{t+1} \leq d_{t}$.

If $d_{t} \leq \alpha /(1-\theta+\alpha \theta)$, then

$\theta\left(1-\alpha_{t+1}\right) d_{t}+\alpha_{t+1} \leq \theta\left(1-\alpha_{t+1}\right) \alpha /(1-\theta+\alpha \theta)+\alpha_{t+1} \leq$
$\leq \theta(1-\alpha) \alpha /(1-\theta+\alpha \theta)+\alpha=\alpha /(1-\theta+\alpha \theta)\left(\right.$ using again that $\left.\alpha>\alpha_{t+1}\right)$.

So $d_{t+1} \leq \theta d_{t}\left(1-\alpha_{t+1}\right)+\alpha_{t+1} \leq \alpha /(1-\theta+\alpha \theta)$.

Thus, either $d_{t} \leq \alpha /(1-\theta+\alpha \theta)$ for all sufficiently large $t$ (which implies the inequality $d_{\infty} \leq \alpha /(1-\theta+\alpha \theta)$ which we are proving), or the sequence $\left\{d_{t}\right\}$ is monotone for all $t>t_{0}$ and consequently, $d_{t} \rightarrow d_{\infty}$.

In the last case, for any limit point $\alpha_{0}$ of the sequence $\left\{\alpha_{t}\right\}$ (of course, $\alpha_{0} \leq \alpha_{\infty} \leq \alpha$ ) we have:

$$
d_{\infty}=\max \left(d_{\infty}\left(1-\alpha_{0}+\alpha_{0} \theta\right), \theta d_{\infty}\left(1-\alpha_{0}\right)+\alpha_{0}\right) .
$$

When $\alpha_{0} \neq 0$, it follows that $d_{\infty}=\theta d_{\infty}\left(1-\alpha_{0}\right)+\alpha_{0}$, hence $d_{\infty}=$ $\alpha_{0} /\left(1-\theta+\alpha_{0} \theta\right)$ and $\alpha_{0}=\alpha_{\infty}$.

Therefore only the following case remains: $d_{t} \rightarrow d_{\infty}$ and $\alpha_{t} \rightarrow 0$. We have to prove now that $d_{\infty}=0$. Assuming that $d_{\infty} \neq 0$, we obtain that $d_{\infty}=0$ (a contradiction) as follows. For all sufficiently large $t$,

$$
\begin{aligned}
& d_{t+1}:=\max \left(d_{t}\left(1-\alpha_{t+1}+\alpha_{t+1} \theta\right), \theta d_{i}\left(1-\alpha_{t+1}\right)+\alpha_{t+1}\right)= \\
& =d_{t}\left(1-\alpha_{t+1}+\alpha_{t+1} \theta\right) \geq \theta d_{t}\left(1-\alpha_{t+1}\right)+\alpha_{t+1}
\end{aligned}
$$

herice

$$
d_{\infty}=d_{t} \Pi_{s=t+1}^{\infty}\left(1-\alpha_{s}(1-\theta)\right)=0,
$$

because the series $\sum \alpha_{t}$ diverges.

(b) By the definition of $d_{t}$, wc have

$$
d_{t+1} \geq d_{t}\left(1-\alpha_{t+1}+\alpha_{t+1} \theta\right),
$$

hence

$$
d_{t} \geq d_{s} \Pi_{i=s+1}^{\infty}\left(1-\alpha_{i}(1-\theta)\right)=: c_{s}
$$

whenever $t \geq s$, and $c_{s+1}>c_{s}$ for all $s$. Therefore

$$
d_{\infty}:=\limsup d_{t} \geq \limsup c_{t}=\sup _{0 \leq t<\infty} c_{t}=: c_{\infty} .
$$

Since $\sum \alpha_{s}$ converges, $c_{s}>0$ for sufficiently large $s$ and $d_{s} / c_{s} \rightarrow 1$. So $d_{\infty}=c_{\infty}$.

(c) By the definition of $d_{t}$,

$$
d_{t+1} \geq \theta d_{t}\left(1-\alpha_{t+1}\right)+\alpha_{t+1} \geq \alpha_{t+1}, \text { hence } d_{\infty} \geq \alpha_{\infty} .
$$


Suppose now that $\alpha_{t} \rightarrow \alpha_{\infty}>0$. In the view of (a), it remains to prove only that

$$
\liminf d_{t} \geq \alpha_{\infty} /\left(1-\theta+\theta \alpha_{\infty}\right)
$$

We pick any $\alpha<\alpha_{\infty}$ and want to show that

$$
\liminf d_{t} \geq \alpha /(1-\theta+\theta \alpha)
$$

Pick $t_{0}$ such that $\alpha_{t} \geq \alpha$ for all $t \geq t_{0}$. For $t \geq t_{0}$, if $d_{t}<\alpha /(1-\theta+\theta \alpha)$, then $d_{t} \theta\left(1-\alpha_{t+1}\right)+\alpha_{t+1} \geq d_{t}$, hence $d_{t+1} \geq d_{t}$.

On the other hand, if $d_{t} \geq \alpha /(1-\theta+\theta \alpha)$ for some $t$, then

$$
d_{t} \theta\left(1-\alpha_{t}\right)+\alpha_{t} \geq \alpha /(1-\theta+\theta \alpha) \text {, hence } d_{t+1} \geq \alpha /(1-\theta+\theta \alpha) .
$$

Thus, either $d_{t} \geq \alpha /(1-\theta+\theta \alpha)$ for sufficiently large $t$, which implies the wanted inequality $\lim \inf d_{t} \geq \alpha /(1-\theta+\theta \alpha)$, or the sequence $\left\{d_{t}\right\}$ is monotone (non-decreasing).

In the last case, $d_{t} \rightarrow d_{\infty} \leq \alpha /(1-\theta+\theta \alpha)<\alpha_{\infty}\left(1-\theta+\theta \alpha_{\infty}\right)$, which contradicts to equality $d_{\infty}=\max \left(d_{\infty}\left(1-\alpha_{0}+\alpha_{0} \theta\right), \theta d_{\infty}\left(1-\alpha_{0}\right)+\alpha_{0}\right)$ with $\alpha_{0}=\alpha_{\infty}$ observed in (a) above.

(d) Among those $w$ in $[x, y]$ where the function $f$ reaches its minimal value, i.e. $f(w)=f(X)$, we take the point $x^{\prime}$ closest to $z$.

Replacing, if necessary, the interval $[x, y]$ by a subinterval centered around $x^{\prime}$, we assume that $x^{\prime}=x / 2+y / 2, f(u)-f(X) \leq F$ for all $u$ in $[x, y]$ and $f((1-\alpha) x+\alpha y)>f(X)$ whenever $1 / 2<\alpha \leq 1$.

When the condition (b) holds, i.e. $\sum \alpha_{t}<\infty$, our procedure will take place in the interval $\left[x^{\prime}, y\right]$. Note that $f\left(x^{\prime}\right)=f\left(\left[x^{\prime}, y\right]\right)=f(X)$ and $f(u)>f(X)$ for $u \neq x^{\prime}$ in the interval $\left[x^{\prime}, y\right]$. Let $s$ be the smallest $t_{0}$ such that $\alpha_{t}<1$ for all $t \geq t_{0}$. If $s=1$, we take $x_{0}=y$. Otherwise, $\alpha_{s-1}=1$, and we set $x_{0}=y_{t}=x^{\prime}$ for $t<s$ and $y_{s}=y$. In both cases, $x_{s-1}=y$. We set $y_{t}=x^{\prime}$ for $t>s$. Then the condition (2) holds for all $t$ with $\theta=0$, and for all $t \geq s$ we have

$$
x_{t}-x^{\prime}=\Pi_{i=s}^{t}\left(1-\alpha_{i}\right)\left(z-x^{\prime}\right) \text {, so } x_{t} \rightarrow x_{\infty}=x^{\prime}+\prod_{i=s}^{\infty}\left(1-\alpha_{i}\right)\left(z-x^{\prime}\right) \neq x^{\prime}
$$

Thus, $f\left(x_{t}\right) \rightarrow f\left(x_{\infty}\right)>f(X)$.

If the condition (c) holds, i.e. Iim sup $\alpha_{t}=\alpha_{\infty}>0$, then we pick a sequence $\{s(i)\}$ of natural numbers such that: $s(i)<s(i+1)$,

$$
\frac{\alpha_{\infty}}{2} \leq \alpha_{s(i)} \leq \frac{3 \alpha_{\infty}}{2} \text {, and } \prod_{t=y(i)+1}^{s(i+1)-1}\left(1-\alpha_{t}\right) \leq \frac{1}{3} \text { for all } i
$$

We set $x_{0}=x^{\prime}=y_{t}$ for all $t$ outside $\{s(i)\}, y_{s(2 i+1)}=y$, and $y_{s(2 i)}=x$ for all $i$. Then $x_{t}=\beta_{t} y+\left(1-\beta_{t}\right) x$, where: $\beta_{t}=1 / 2$ when $t<s(1) ; \beta_{s(1)}=$ $\left(1+\alpha_{s(t)}\right) / 2 ; 1 / 2<\beta_{t}$ when $s(1) \leq t<s(2)$;

$$
\begin{aligned}
\beta_{s(2)-1} & =\frac{1}{2}+\frac{\alpha_{s(1)}}{2} \Pi_{t=s(1)+1}^{s(2)-1}\left(1-\alpha_{t}\right) \leq \frac{1}{2}+\frac{\alpha_{\infty}}{4} \\
\beta_{s(2)} & =\beta_{s(2)-1}\left(1-\alpha_{s(2)}\right) \leq\left(1-\alpha_{\infty} 2 / 4\right) / 2 .
\end{aligned}
$$


Induction on $i$ shows:

$1 / 2 \leq \beta_{t} \leq\left(1+3 \alpha_{\infty} / 2\right) / 2$ for $s(3 i-1) \leq t<s(2 i)$ and $\beta_{s(2 i-1)} \leq$

$1 / 2+\alpha_{\infty} / 4,\left(1+3 \alpha_{\infty} / 2\right) / 2 \leq \beta_{t} \leq 1 / 2$ for $s(2 i) \leq t<s(2 i+1)$ and

$\beta_{s(2 i+1)-1} \geq \geq 1 / 2-\alpha_{\infty} / 4$.

So $\alpha_{\infty}^{2} / 8 \leq\left|\beta_{s(i)}-1 / 2\right| \leq 3 \alpha_{\infty} / 4$ for all $i$.

Therefore $\lim \sup \beta_{t} \geq 1 / 2+\alpha_{\infty} 2 / 8>1 / 2$, hence limsup $f\left(x_{t}\right)>f\left(x^{\prime}\right)=$ $f(X)$.

Thus, $f\left(x_{t}\right)$ does not converge to $f(X)$.

Remark. If $X$ does not contain any interval $[x, y]$ with $x \neq y$, then, evidently, $x_{t}=x_{0}$ for all $t$ and any procedure (1); moreover, the condition (2) with $t=1$ implies that $f\left(x_{0}\right)=f(X)$.

Example. Let $\theta=0$ in Theorem 1. When $\alpha_{2}=1 /(t+1)$ for all $t$, then $d_{t}=1 /(t+1)$ for all $t$. In the next section we will see that this $\left\{\alpha_{t}\right\}$ is the best sequence when $\theta=0$.

\section{The best damping sequence}

Now we want to find the best damping sequence $\left\{\alpha_{t}\right\}$, that is, the one which gives the minimal value for $d_{T}$ (when $T$ is finite) in Theorem 1. The following theorem claims, among other things, the existence and uniqueness of such a sequence and its independence on $F$ and $T$ (when $T$ incrcases, new members are added to the sequence, but old members stay the same).

Theorem 3. For a fixed $\theta$ in the interval $[0,1]$ let us define inductively a sequence $\left\{A_{t}\right\}=\left\{A_{t}(\theta)\right\}$ by $A_{1}=1 / 2$ and

$$
A_{t+1}=A_{t}\left(1-A_{t}+\theta A_{t}\right) /\left(1-(1-2 \theta) A_{\ell}^{2}\right) \text { for } t \geq 1 \text {. }
$$

Then:

(a) the sequence $\left\{A_{t}(\theta)\right\}$ strictly decreases to 0 ; when $\theta<1$ we have

$$
A_{t}(\theta)=(1-\theta) /\left((t+1)(1-\theta)^{2}+\theta^{2} \ln (t)+\theta c_{t}(\theta) /(1-\theta)^{2}\right)
$$

with $-7 \leq c_{t}(\theta) \leq 3$ for all t and $\theta$; when $\theta=1$ we have

$$
A_{t}(1)=\left(3 / A_{t}(1 / 3)-2\right)^{-1 / 2}=\left(2 t+\ln (t) / 2+27 c_{t}(1 / 3) / 8\right)^{-1 / 2} ;
$$

(b) the sequence $D_{t}(\theta):=A_{t+1}(\theta) /\left(1-\theta+A_{t+1}(\theta)(2 \theta-1)\right)$ decreases strictly to 0 and has the form

$$
D_{t}(\theta)=\left((1-\theta)^{2} t+\theta^{2} \ln (t+1)-2 \theta+2 \theta^{2}+1+\theta c_{t}(\theta) /(1-\theta)^{2}\right)^{-1}
$$

when $\theta<1$; otherwise, $D_{t}(1)=1$ for all $t$; 
(c) for any $L, X, f, T, \theta, F$ as above and any procedure (1),(2),(4), with $\left\{\alpha_{t}\right\}=A_{t}(\theta)$ we have

$$
f\left(x_{t}\right)-f(X) \leq F D_{t}(\theta), 0 \leq t<T+1
$$

(d) for arbitrary $T, F>0, \theta<1,\left\{\alpha_{t}\right\} \in[0,1]^{T}$ there exists $L, X, f$, and a procedure (1),(2),(4) such that $f\left(x_{t}\right)-f(X) \geq F D_{t}(\theta)$ for all $t$ in the interval $0 \leq t<T+1$, and the inequality is strict for each $t$ such that $\alpha_{s} \neq$ $A_{s}(\theta)$ for some $s \leq t$.

Proof: (a) Assume that for some $t$ we have shown that $0<A_{t} \leq 1 / 2$ (for $t=1$, this is the case), and let us show that then $0<A_{t+1}<A_{t}$. Indeed the inequalities $0<A_{t} \leq 1 / 2$ and $0 \leq \theta \leq 1$ imply that $1>(1-\theta) A_{t}>(1-2 \theta) A_{t}^{2}$, hence

$$
0<A_{t+1} / A_{t}=\left(1-A_{t}(1-\theta)\right) /\left(1-A_{t}^{2}(1-2 \theta)\right)<1 \text {, so } 0<A_{t+1}<A_{t} \text {. }
$$

Let $A_{\infty}$ be the limit of the monotone sequence $\left\{A_{t}\right\}$. Then $A_{\infty} \geq 0$ and

$$
A_{\infty}=A_{\infty}\left(1-A_{\infty}(1-\theta)\right) /\left(1-A_{\infty}^{2}(1-2 \theta)\right),
$$

hence $A_{\infty}=0$, because $1-\alpha(1-\theta)<1-\alpha^{2}(1-2 \theta)$ for any $\alpha$ in the interval $0<\alpha \leq 1 / 2$.

Let us now find the asymptotic of the sequence $\left\{A_{t}\right\}$ when $\theta<1$. Set $x_{t}=1 / A_{t}$. Then $x_{1}=2 \leq x_{t}$ for all $t$, and the defining equality for $A_{t}$ takes the form

$$
x_{t+1}=x_{t}+(1-\theta)+\theta^{2} /\left(x_{t}-1+\theta\right) .
$$

This can be rewritten as follows:

$$
y_{0}=\theta_{1} y_{t+1}=y_{t}+\theta^{2} /\left((1-\theta) t+y_{t}\right)
$$

where

$$
y_{t}:=x_{t}-(t+1)(1-\theta), y_{1}=2 \theta, y_{2}=\theta(2+3 \theta) /(1+\theta) \text {. }
$$

It is clear from (5) that $y_{t} \geq 0$ for all $t$ and that $y_{t+1}-y_{t} \leq \theta^{2} /(1-\theta) t$. So for $t \geq 2$ we obtain:

$$
\begin{aligned}
& y_{t}=\sum_{s=3}^{t}\left(y_{s}-y_{s-1}\right)+y_{2} \leq \frac{\theta^{2}}{1-\theta} \sum_{s=2}^{t-1} 1 / s+\frac{\theta(2+3 \theta)}{1+\theta} \leq \\
& \leq \frac{\theta^{2}}{1-\theta} \int_{1}^{t-1} \frac{d t}{t}+3 \theta=\frac{\theta^{2}}{1-\theta} \ln (t-1)+3 \theta \leq \frac{\theta}{1-\theta}(\ln (t)+3) .
\end{aligned}
$$

The obtained inequality $y_{t} \leq \theta(\ln (t)+3) /(1-\theta)$ holds also for $t=1$. 
On the other hand, substituting this upper bound for $y_{t}$ into (5), we obtain that

$$
\begin{aligned}
& y_{t+1}-y_{t}=\theta^{2} /\left((1-\theta) t+y_{t}\right)=\theta^{2} /(1-\theta) t-\theta^{2} y_{t} /(1-\theta) t\left((1-\theta) t+y_{t}\right) \geq \\
& \geq \theta^{2} /(1-\theta) t-\theta^{3}(\ln (t)+3) / t^{2}(1-\theta)^{3} .
\end{aligned}
$$

So, for any $t \geq 1$, we have:

$$
\begin{gathered}
y_{t} \geq 2 \theta+\sum_{s=1}^{t} \frac{\theta^{2}}{s(1-\theta)}-\theta^{3} \sum_{s=1}^{t} \frac{\ln (s)+3}{s^{2}(1-\theta)^{3}} \geq \\
\geq 2 \theta+\theta^{2} \int_{1}^{t} \frac{d s}{s(1-\theta)}-\frac{3 \theta^{3}}{(1-\theta)^{3}}-\theta^{3} \int_{1}^{t} \frac{\ln (s)+3}{s^{2}(1-\theta)^{3}}= \\
=2 \theta+\theta^{2} \ln (t) /(1-\theta)-3 \theta^{3} /(1-\theta)^{3}+\theta^{3}((\ln (t)+4) / t-4) /(1-\theta)^{3} \geq \\
\geq \theta^{2} \ln (t) /(1-\theta)-7 \theta^{3} /(1-\theta)^{3} .
\end{gathered}
$$

Therefore, for

$$
c_{t}(\theta):=(1-\theta)^{3}\left(y_{t}-\theta^{2} \ln (t) /(1-\theta)\right) / \theta
$$

we obtain that

$$
\begin{aligned}
c_{t}(\theta) & \leq(1-\theta)^{3}\left(\theta^{2} \ln (t-1) /(1-\theta)+3 \theta-\theta^{2} \ln (t) /(1-\theta) / \theta \leq\right. \\
& \leq(1-\theta)^{3}(3 \theta) / \theta=3(1-\theta)^{3} \leq 3
\end{aligned}
$$

and

$$
c_{t}(\theta) \geq(1-\theta)^{3}\left(-7 \theta^{3} /(1-\theta)^{3}\right) / \theta=-7 \theta^{4} \geq-7 .
$$

When $t=1$, we have

$$
c_{1}(\theta)=(1-\theta)^{3}(2 \theta) / \theta=2(1-\theta)^{3} .
$$

Thus, $-7 \leq c_{t}(\theta) \leq 3$ for all $t$ and $\theta$, hence $\left|c_{t}(\theta)\right|$ is bounded uniformly over all $t$ and $\theta$.

Let us find now $A_{t}(1)$ in the terms of $A_{t}(1 / 3)$. By the definition, $A_{1}=1 / 2$ and $A_{t+1}(1)=A_{t}(1) /\left(1+A_{t}(1)^{2}\right)$ or

$$
\frac{1}{\sqrt{y_{t+1}}}=\frac{\sqrt{y_{t}}}{1+y_{t}}
$$

where $y_{t}:=A_{t}(1)^{-2}$. Therefore

$$
y_{1}=4 \text { and } y_{t+1}=y_{t}+2+1 / y_{t} \text { for } t \geq 1 .
$$


On the other hand, for $x_{t}:=1 / A_{t}(1 / 3)$ we have

$$
x_{1}=2 \text { and } x_{t+1}=x_{t}+2 / 3+1 /\left(9 x_{t}-6\right) \text { for } t \geq 1 .
$$

So for $z_{t}:=3 x_{t}-2$ we obtain that

$$
z_{1}=4 \text { and } z_{t+1}=z_{t}+2+1 / z_{t} \text { for } t \geq 1 .
$$

Thus, $z_{t}=y_{t}$ for all $t \geq 1$, i.e.

$$
1 / A_{t}(1)=\left(3 / A_{t}(1 / 3)-2\right)^{1 / 2}=\left(2 t+\ln (t) / 2+27 c_{t}(1 / 3) / 8\right)^{1 / 2} .
$$

(b) This follows from the definition of $D_{t}$ and the part (a).

(c) By the definition,

$$
D_{t}:=D_{t}(\theta)=A_{t+1} /\left(1-\theta+2 \theta A_{t+1}-A_{t+1}\right) .
$$

Expressing here $A_{t+1}=A_{t+1}(\theta)$ in terms of $A_{t}=A_{t}(\theta)$ and using the definition of $D_{t-1}$, we obtain:

$$
\begin{aligned}
D_{t} & =1 /\left((1-\theta) / A_{t+1}+2 \theta-1\right)=A_{t}\left(1-A_{t}+\theta A_{t}\right) /\left(1-\theta+(2 \theta-1) A_{t}\right)= \\
& =D_{t-1}\left(1-A_{t}+\theta A_{t}\right)=\theta D_{t-1}\left(1-A_{t}\right)+A_{t}= \\
& =\max \left(D_{t-1}\left(1-A_{t}+\theta A_{t}\right), \theta D_{t-1}\left(1-A_{t}\right)+A_{t}\right) .
\end{aligned}
$$

Moreover, $D_{0}(\theta)=1$, since $A_{1}(\theta)=1 / 2$.

Therefore the sequence $\left\{d_{t}\right\}:=\left\{D_{t}(\theta)\right\}$ coincides with the sequence $\left\{d_{t}\right\}$ of Theorem 1 when $\alpha_{t}$ is taken to be $A_{t}(\theta)$ for all $t$. So the conclusion of Theorem $3(\mathrm{c})$ with $\theta<1$ follows from Theorem 1 . When $\theta=1$ the conclusion coincides with the condition (4).

vglue 0.3 true cm (d) Let us define $L, X, f, y_{t}, x_{t}$ as in the proof of Theorem 1. Then $f\left(x_{t}\right)-f(X)=F d_{t}$, where $d_{0}=1$ and

$$
d_{t+1}:=\max \left\{d_{t}\left(1-\alpha_{t+1}+\alpha_{t+1} \theta\right), \theta d_{i}\left(1-\alpha_{t+1}\right)+\alpha_{t+1}\right) \text { for } t \geq 0 \text {. }
$$

We want to prove now that $d_{t} \geq D_{t}(\theta)$ for all $t$, and that this inequality is strict for each $t$ such that $\alpha_{s} \neq A_{s}(\theta)$ for some $s \leq t$.

Proceeding by induction on $t$, we assume, for some $t$, that $d_{t} \geq D_{t}(\theta)$ and in the case of equality every $\alpha_{s}$ with $s \leq t$ coincides with $A_{s}(\theta)$.

If $\alpha_{t+1} \leq A_{t+1}(\theta)$, then

$$
d_{t+1} \geq d_{t}\left(1-(1-\theta) \alpha_{t+1}\right) \geq D_{t}(\theta)\left(1-(1-\theta) \alpha_{t+1}\right)=D_{t+1}(\theta) .
$$

Otherwise, i.e. when $\alpha_{t+1}>A_{t+1}(\theta)$, we have

$$
d_{t+1} \geq \theta d_{t}\left(1-\alpha_{t+1}\right)+\alpha_{t+1} \geq \theta D_{t}(\theta)\left(1-A_{t+1}(\theta)\right)+A_{t+1}(\theta)=D_{t+1}(\theta) \text {. }
$$

Thus, $d_{t} \geq D_{t}(\theta)$ in both cases. Furthermore, the equality $d_{t+1}=D_{t+1}(\theta)$ implies, evidently, that $d_{t}=D_{t}(\theta)$ and $\left.\alpha_{t+1}=A_{t+1}(\theta)\right)$.

Remark. For any $\varepsilon>0$, an example can be constructed (see the remark in the previous section) with a convex $f$ defined on the whole $L$ and a convex $X$ such that $f\left(x_{t}\right)-f(X) \geq F\left(D_{t}(\theta)-\varepsilon\right)$ for all $t$ in the interval $0 \leq t<T+1$, and $f\left(x_{t}\right)-f(X)>F D_{t}(\theta)$ for each $t$ such that $\alpha_{s} \neq A_{s}(\theta)$ for some $s \leq t$. So the sequence $\left\{A_{t}(\theta)\right\}$ stays the best in this restricted class of functions $f$.

Acknowlegment. A referee made many corrections in this paper. 


\title{
References
}

1. V.Z.BELEN'KY, V.A.VOLKONSKY,S.A.IVANKOV,A.B.POMANSKY, A. D. SHAPIRO, "Iterative methods in game theory and programming ," Nauca, Moscow, 1974. (in Russian)

2. G. W. BROWN, Some notes on computation of game solutions, RAND Report P-78, (April 1949), The Rand Corporation, Santa Monica, California.

3. M. M. DAY, "Normed linear spaces," Springer-Verlag, 1958.

4. B. T. POLJAK, A general method for solving extremum problems, Soviet Math. Dokl. 8 (1967), 593-597.

5. J. RoBINSON, An iterative method of solving a game, Ann. Math. 54 (1951), 246-301.

\author{
Department of Mathematics \\ Perm State University \\ University Park, PA 16802, USA \\ and \\ Institut d'Estudis Catajans \\ Centre de Recerca Matcmàtica \\ Apartat 50-08193 Bellaterra, SPAIN.
}

Rebut el $1 \gamma$ de Maig de 1988 